\title{
Effect of abdominal muscle training on respiratory muscle strength and forced expiratory flows in sedentary, healthy adolescents
}

a. Exercise Biology Lab (Laboratorio de Biología del Ejercicio, LBE), School of

Kinesiology, School of Health Sciences,

Universidad San

Sebastián, Concepción, Chile.

b. Master Program in Cardiorespiratory

Kinesiology, School of Kinesiology, School of Health Sciences, Universidad San

Sebastián, Concepción, Chile.

c. School of Kinesiology, School of Health, Universidad Santo Tomás, Concepción, Chile.

d.Research Task Force in Cardiovascular and Respiratory Health IDEAS-CVR, Concepción, Chile.

e. Doctoral Program in Medical Sciences, School of Medicine, Universidad de La Frontera, Temuco, Chile.

f. Department of Surgery and Center of Excellence for Morphological and Surgical Studies (Centro de Excelencia en Estudios

Morfológicos y Quirúrgicos, CEMyQ), Universidad de $\mathrm{La}$ Frontera, Chile.

g. Center for Bioscience Research, Universidad Autónoma de Chile, Chile.

E-mail address:

Iván Rodríguez Núñez, Kinesiologist, ivan.rodriguez@uss.cl

\section{Funding:}

This study was funded by the School of Kinesiology of Universidad San Sebastián, Concepción, Chile.

Conflict of interest: None.

\author{
Iván Rodríguez-Núñez, Kinesiologista,b,c,de, Ximena Navarro, Kinesiologist ${ }^{b}$, \\ Darwin Gatica, Kinesiologist ${ }^{c, d e}$ and Carlos Manterola, M.D. ${ }^{e, f, g}$
}

\section{ABSTRACT}

Introduction. Respiratory muscle training is the most commonly used method to revert respiratory muscle weakness; however, the effect of protocols based on non-respiratory maneuvers has notbeen adequately studied in the pediatric population. The objective of this study was to establish the effect of abdominal muscle training on respiratory muscle strength and forced expiratory flows in healthy adolescents. Methods. This was a quasi-experiment. The sample was made up of healthy adolescents divided into two groups: an experimental group who completed eight weeks of active abdominal muscle training, and an equivalent control group. The following indicators were measured: abdominal muscle strength, maximal inspiratory pressure, maximal expiratory pressure (MEP), peak expiratory flow, and peak cough flow, before and after protocol completion. A value of $p<0.05$ was considered significant.

Results. All studied outcome measures increased significantly in the experimental group but only MEP increased in the control group. In addition, a higher MEP was observed in the experimental group compared to the control group at the end of the protocol, together with a greater increase in MEP and peak expiratory flow. Finally, the increase in MEP was associated with an increase in cough peak flow in the experimental group but not in the control group.

Conclusion. After eight weeks of abdominal muscle training, MEP and peak expiratory flow increased in healthy (sedentary) adolescents. Such effects were associated with interventioninduced increases in cough peak flow.

Key words: respiratory muscle training, peak expiratory flow, respiratory muscle, abdominal training, cough.

http:/ /dx.doi.org/10.5546/aap.2016.eng.434

\section{INTRODUCTION}

Respiratory muscle weakness is one of the major functional consequences suffered by children with chronic respiratory disease (CRD). ${ }^{1-3}$ It leads to alveolar hypoventilation, microatelectases, and failure of the cough mechanism, and these factors increase the risk for respiratory failure. ${ }^{4}$
It has been observed that respiratory muscle training (RMT) may be effective to mitigate the deterioration in muscle strength and endurance, ${ }^{5-7}$ and peak cough flow (PCF) in children with neuromuscular disorders. ${ }^{6}$ However, there is still no consensus on which are the most effective training protocols to achieve function targets. ${ }^{8}$ In the clinical setting, RMT using a threshold valve (Threshold ${ }^{\circledR}$ ) is the most widely used modality; ${ }^{7}$ however, it is an indirect and non-specific method to achieve muscle strengthening.

From a physiological perspective, the performance of respiratory muscles is affected by both chest and abdominal muscles. Thus, it has been demonstrated that several exercise modalities focused on abdominal muscles and upper and lower limbs stimulate the diaphragm, increase transdiaphragmatic pressure, and induce different levels of abdominal muscle fatigue. ${ }^{9,10}$

This precedent underscores the potential effect of training protocols based on non-respiratory exercises on muscle strength and pulmonary function parameters. Hence, several studies conducted in adults have demonstrated that non-respiratory maneuvers, e.g. sit-ups, may increase respiratory muscle strength. ${ }^{11}$ In addition, it has been observed that abdominal muscle training protocols, both passive (based on electrical stimulation $)^{12,13}$ and active, may be effective to improve respiratory muscle strength and forced expiratory flows. ${ }^{14}$ However, the effect of this type of training strategy has not been adequately studied in the pediatric population. ${ }^{14}$ 
Therefore, the goal of this study was to assess the effect of abdominal muscle training on respiratory muscle strength and forced expiratory flows in sedentary, healthy adolescents.

\section{MATERIAL AND METHODS}

This was a quasi-experimental study with an equivalent control group. Preliminary results from the experimental group were reported in a previously published pilot study. ${ }^{14}$

Sample: Healthy adolescents attending a public school from the city of Concepción, Chile were selected by convenience, with no gender restrictions. Inclusion criteria included sedentary, healthy adolescents (who did physical activity for less than 60 minutes twice a week) and who had not done any muscle training in the past month. Exclusion criteria were the presence of cardiorespiratory or musculoskeletal disease and smoking. Eligibility criteria were verified during an interview with every study participant, and using a checklist completed by parents before their children were enrolled in the study. No cardiovascular health checks, such as an echocardiogram or stress test, were done before the experimental protocol. Both study participants and their parents signed an informed assent and consent, respectively, and the study was authorized by the Institutional Ethics Committee. The study was conducted between June 2014 and May 2015.

The sample size was estimated based on the previously published methodology. ${ }^{14}$ An $80 \%$ statistical power and a 5\% type I error were considered; therefore, the minimum sample needed for this study was 17 subjects per group. In this context, the experimental group was first selected as per inclusion criteria, and then the control group was established. Both groups were paired based on biodemographic characteristics (weight, height, age, and gender) and pulmonary function outcome measures (respiratory muscle strength and forced expiratory flows) before the protocol.

Outcome measures: Age, gender, height, and body weight were recorded before study initiation. Weight and height were measured using an analog scale and a tape measure, and expressed in kilograms $(\mathrm{kg})$ and centimeters $(\mathrm{cm})$, respectively.

Outcome measures of interest included respiratory muscle strength and forced respiratory flows. Respiratory muscle strength was established based on maximal inspiratory pressure (MIP), measured during a maximum inspiratory effort, from residual volume, and maintained for at least one second, and maximal expiratory pressure (MEP), measured during a maximum expiratory effort, from total lung capacity, and maintained for at least one second. An aneroid vacuum/pressure gauge NS 120TRS, calibrated in centimeters of water (from 0 to -120 , and from 0 to $+120 \mathrm{cmH}_{2} \mathrm{O}$ ) was used to take measurements. MIP and MEP values were expressed as absolute values $\left(\mathrm{cmH}_{2} \mathrm{O}\right) \cdot{ }^{15}$

Also, both peak expiratory flow (PEF) and peak cough flow (PCF) were measured using a flow meter (Mini-Wright ${ }^{\circledR}$; Clement Clarke International, Essex, England). PEF was measured with the subject standing and wearing a nose clip, and the indication was given to blow with the greatest possible force from total lung capacity. The test was performed at least three times and a maximum of eight times; the highest reproducible value in three attempts with a difference no greater than $10 \%$ between each value was recorded. ${ }^{16}$ PCF was measured with the subject seated, and the indication was given to inhale as deeply as possible and then to perform a maximum coughing effort through the flow meter. Results were expressed in liters/minute. ${ }^{17}$

In addition, as a measure of effectiveness of abdominal muscle training (AMT), abdominal muscle strength (AMS) was established. AMS was measured using the sit-up test, a functional test that has been widely used in both healthy subjects and patients with chronic respiratory disease. ${ }^{14,18}$ The subject initially lies supine with knees bent at $90^{\circ}$, feet on the floor, and hands on the side of the head. An assistant helps the subject keep his/her feet on the mat. At this time, the examiner gives the indication to start the test, which consists in performing the maximum number of trunk flexion-extension repetitions in 30 seconds.

Training protocol: AMT was conducted as per Rodríguez et al. ${ }^{14}$ Briefly described, the protocol consisted in training each abdominal muscle group during eight weeks. During each exercise session, all muscle groups were trained actively, with no external aid, reaching between 200 and 300 repetitions per session. Sessions took place twice weekly, each lasting 45 minutes. On their side, subjects in the control group were asked to do their normal activities and were monitored during the eight weeks that the experimental protocol lasted. In the end, both pulmonary function and AMS were measured one day before and one day after the training protocol in both study groups. 
Analysis schedule: The MedCalc Statistical Software, version 14.12.0 (MedCalc Software bvba, Ostend, Belgium; http:/ /www.medcalc.org, 2014) was used to do an exploratory data analysis, and normality was measured using the Shapiro-Wilk test. Once the normal distribution of data was verified, descriptive statistics were established using average and standard error of the mean (SEM).

The absolute value of pulmonary function parameters was compared between study groups using the $t$ test for independent samples and also for paired samples, to assess differences between absolute values before and after the intervention.

In addition, the Mann-Whitney $U$ test was used to compare the size of variations in outcome measures of interest between the control and the experimental groups. The association between dichotomous outcome measures was assessed using a $\chi^{2}$ test.

Finally, a linear regression analysis was done to establish the association between the size of the effect on MEP and forced expiratory flows (PEF and PCF).

A value of $p<0.05$ was considered significant.

\section{RESULTS}

General characteristics of the sample: The experimental group was made up of 17 subjects ( 8 boys) and the control group included 23 subjects ( 9 boys). Age, gender and anthropometric characteristics are detailed in Table 1, and were similar in both study groups.

Also, no significant difference was observed in terms of respiratory muscle strength (MIP and MEP), forced expiratory flows (PEF and PCF), and AMS between groups before starting the training protocol (Table 2).

Effect of training: All subjects completed the protocol without incidents. No subject from either group was excluded from the protocol due to non-adherence or health conditions. Following the training program, only subjects in the experimental group increased the number of sit-ups done in 30 seconds by $21.7 \%(p=0.0001)$.

Both respiratory muscle strength and forced expiratory flows increased significantly from baseline in the experimental group. Thus, MIP increased $16.8 \%(p=0.006)$ and MEP , 50.2\% $(p<0.0001)$. PEF rose $8.4 \%(p=0.003)$ and PCF, $9.1 \%(p=0.015)$. On their side, these outcome

TABLE 1. Baseline characteristics of the sample

\begin{tabular}{lccc}
\hline Outcome measures & Control group $(\mathbf{n}=\mathbf{2 3})$ & Experimental group $(\mathbf{n}=\mathbf{1 7})$ & $\boldsymbol{P}$ value \\
\hline Gender $(\mathrm{M} / \mathrm{F})$ & $9 / 14$ & $8 / 9$ & 0.8588 \\
Age $($ years old $)$ & $15.4 \pm 0.1$ & $15.2 \pm 0.1$ & 0.1609 \\
Height $(\mathrm{cm})$ & $161.4 \pm 1.9$ & $162.9 \pm 1.7$ & 0.5601 \\
Weight $(\mathrm{kg})$ & $61.6 \pm 2.3$ & $56.6 \pm 2.4$ & 0.1537 \\
\hline
\end{tabular}

M: male; F: female. Results are described as average \pm standard error of the mean.

TABLE 2. Respiratory muscle strength and forced expiratory flows before and after training

\begin{tabular}{lccc}
\hline Outcome measures & Control group $(\mathbf{n}=\mathbf{2 3})$ & Experimental group $(\mathbf{n}=\mathbf{1 7})$ & $\boldsymbol{P}$ value \\
\hline Pre-MIP $\left(\mathrm{cmH}_{2} \mathrm{O}\right)$ & $99.6 \pm 5.9$ & $96.5 \pm 4.3$ & 0.6828 \\
Post-MIP $\left(\mathrm{cmH}_{2} \mathrm{O}\right)$ & $108.1 \pm 4.1$ & $112.8 \pm 4.3^{* *}$ & 0.4051 \\
Pre-MEP $\left(\mathrm{cmH}_{2} \mathrm{O}\right)$ & $84.5 \pm 3.9$ & $69.4 \pm 3.8$ & 0.0799 \\
Post-MEP $\left(\mathrm{cmH}_{2} \mathrm{O}\right)$ & $93.5 \pm 2.0^{* * *}$ & $104.3 \pm 3.3^{* * *}$ & 0.0125 \\
Pre-PEF $(\mathrm{L} / \mathrm{min})$ & $416.5 \pm 19.9$ & $424.3 \pm 22.6$ & 0.4379 \\
Post-PEF $(\mathrm{L} / \mathrm{min})$ & $424.5 \pm 15.8$ & $457.0 \pm 19.0^{* *}$ & 0.0759 \\
Pre-PCF $(\mathrm{L} / \mathrm{min})$ & $382.2 \pm 18.5$ & $401.2 \pm 19.0$ & 0.4898 \\
Post-PCF $(\mathrm{L} / \mathrm{min})$ & $410.3 \pm 13.7$ & $437.6 \pm 19.2^{*}$ & 0.2520 \\
Pre-sit-up test $\left(\mathrm{n}^{\circ}\right.$ reps/30 s) & $23.3 \pm 1.4$ & $23.5 \pm 1.0$ & 0.4592 \\
Post-sit-up test $\left(\mathrm{n}^{\circ}\right.$ reps/30 s) & $23.4 \pm 1.7$ & $28.6 \pm 0.8^{* * *}$ & 0.0039 \\
\hline
\end{tabular}

MIP: maximal inspiratory pressure, MEP: maximal expiratory pressure, PEF: peak expiratory flow, PCF: peak cough flow, sit-up test: number of repetitions done in 30 seconds.

Results are described as mean \pm standard error of the mean. ${ }^{*}<0.05,{ }^{* *}<0.01,{ }^{* * *}<0.001$. 
measures increased mildly in the control group; only MEP showed a significant rise $(+16 \%$, $p=0.0004$ ).

The inter-group analysis following the intervention showed a greater respiratory muscle strength and higher forced expiratory flows in the experimental group; however, a statistically significant difference was observed only for MEP $(+14.2 \%, p=0.0125)$.

Also, the analysis of the effect size on studied outcome measures indicated, for the experimental group, a greater functional gain across all outcome measures compared to the control group; for the latter, functional gain was statistically significant only in terms of MEP $(p<0.0001)$ and PEF $(p=0.02)$ (Figure 1).

The linear regression analysis of gains in MEP and forced expiratory flows (PEF and PCF) showed a positive correlation between MEP and PCF $(r=0.66, r 2=0.44, p=0.0038)$ in the experimental group. This was not observed in the control group (slope for the experimental group: 2.4, 95\% confidence interval (CI): 0.9-3.9; slope for the control group: $0.7,95 \%$ CI: -1.7 to 3.18 ) (Table 3 - Figure 2). No association was observed between MEP and PEF gains $(p=0.12)$.

\section{DISCUSSION}

The main findings of this study revealed that AMT was effective to increase respiratory muscle strength, especially, expiratory muscle strength (MEP) and PEF. In turn, training-induced MEP gains were associated with increases in cough function in the experimental group.

These results are consistent with those obtained in studies that assessed the effect of non-respiratory maneuvers on respiratory muscle performance. Strongoli et al. demonstrated that maneuvers such as standing and trunk flexion from a sitting and supine position involve the respiratory muscles and significantly increase diaphragmatic pressure to such a level as to induce a training stimulus. ${ }^{9}$ DePalo et al. showed that training biceps and trunk flexion for 16 weeks was associated with significant increases in MIP (+27.6\%) and MEP (+36.9\%). ${ }^{11}$
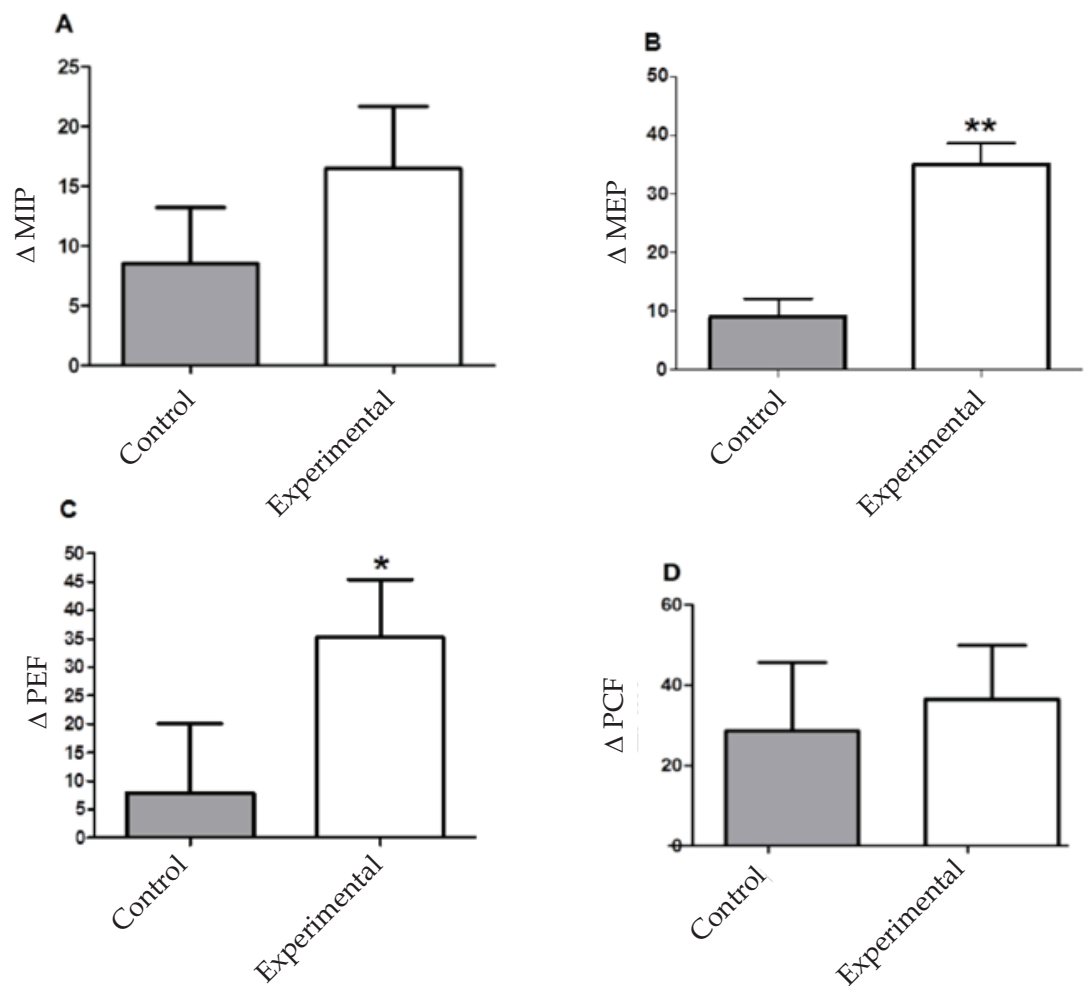

A shows the effect on maximal inspiratory pressure (MIP); B shows the effect on maximal expiratory pressure (MEP); 
Our study evidenced that doing specific exercises to strengthen abdominal muscles would mainly favor muscles involved in active exhalation; however, results were not conclusive regarding the effects on inspiratory muscles.

Similar results have also been reported with conventional respiratory muscle training protocols, which have proven to be effective to improve respiratory muscle strength and physical fitness in healthy, untrained subjects and athletes. ${ }^{19,20}$ In the clinical setting, it has been reported that inspiratory muscle training improves both respiratory muscle strength and endurance in children with neuromuscular disorders, ${ }^{5}$ and even that home-based (unsupervised) training protocols induce increased respiratory muscle strength and have positive effects on force expiratory flows. ${ }^{6}$
In addition, AMT significantly increased PEF and PCF in the experimental group; however, the effect size was meaningfully superior to the control group only in terms of PEF. Nevertheless, a significant association was observed between MEP and PCF gains. In this setting, it has been previously observed that six weeks of abdominal muscle electrical stimulation together with voluntary cough maneuvers may be effective to increase PCF and abdominal and gastric pressures during forced expiration. ${ }^{13}$ On their side, Gollee et al. observed that superficial abdominal muscle functional stimulation increased PCF and tidal volume in tetraplegic subjects. ${ }^{21}$ The linear regression analysis done in our study estimated that per every $\mathrm{cmH}_{2} \mathrm{O}$ of increase in MEP, PCF increased $2.4 \mathrm{~L} / \mathrm{min}$, and this confirms both outcome measures are closely dependent on

TABLE 3. Linear regression analysis between the size of the effect on maximal expiratory pressure and peak cough flow

\begin{tabular}{lccccc}
\hline Group & \multicolumn{1}{c}{ Slope* $^{*}$} & Intercept & r & $\mathbf{r}^{2}$ & $P$ value \\
\hline Experimental group & 2.5 (de 0.9 a 3.9) & -49.5 (de -107.3 a 8.4) & 0.66 (de 0.27 a 0.87) & 0.43 & 0.0038 \\
Control group & 0.72 (de -1.7 a 3.2) & 22.1 (de -20.3 a 64.4) & 0.13 (de -0.29 a 0.51) & 0.02 & 0.5486 \\
\hline
\end{tabular}

Results are described as absolute value and 95\% confidence interval.

FIGURE 2. Linear regression between the size of the effect on peak cough flow and maximal expiratory pressure

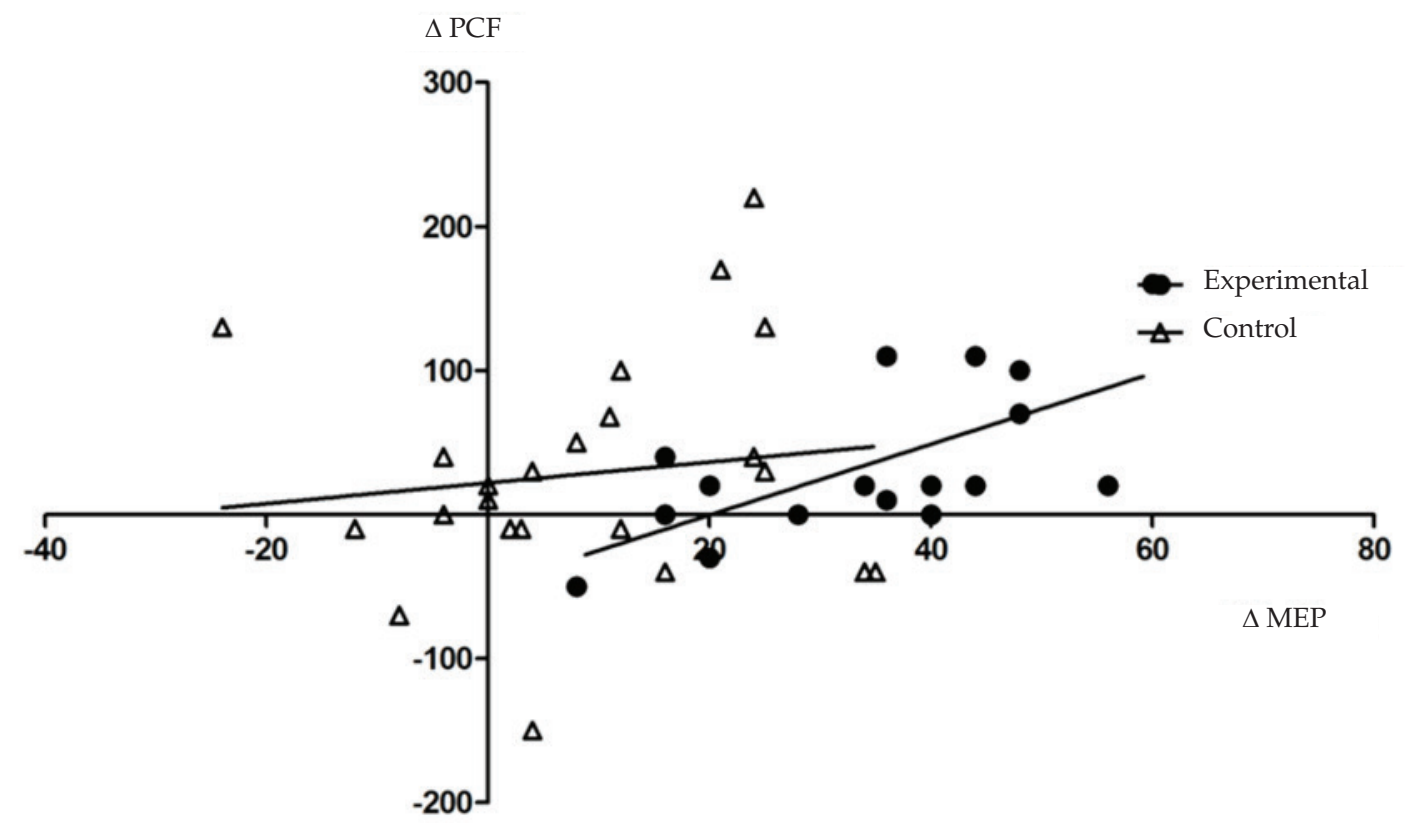

PCF: peak cough flow; MEP: maximal expiratory pressure.

* $Y$ axis outcome measure: gain in peak cough flow induced by training; $X$ axis outcome measure: gain in maximal expiratory pressure induced by training. 
each other. In biomechanical terms, during cough mechanism, expiratory muscles play a critical role both during relaxation in the inspiratory phase and during isometric contraction in the compression phase, which allows raising intraabdominal pressure up to $300 \mathrm{mmHg} .^{22}$

One of the limitations of this study is that arbitrarily establishing an equivalent control group with no randomization may be a potential source of bias. In this context, pairing was attempted based on relevant parameters (anthropometric outcome measures and respiratory function); however, it is not possible to rule out the existence of another uncontrolled co-variable that may affect group homogeneity.

In relation to reliability of outcome measures of interest, the control group observed certain differences in values obtained before and after the intervention protocol, mainly in relation to MEP. In this regard, prior studies have demonstrated different reliability levels in the measurement of respiratory function outcome measures and, specifically, a moderate test-retest agreement in MEP (test-retest difference: $10 \mathrm{cmH}_{2} \mathrm{O}$ ). Moran et al. observed a difference of $10 \mathrm{cmH}_{2} \mathrm{O}$ in $\operatorname{MEP}(p<0.05),{ }^{23}$ which is consistent with what we observed in our study. Nevertheless, the experimental group showed a greater functional gain in MEP compared to the control group. This makes it possible to establish that the increase in expiratory muscle strength observed in the experimental group was the result of the effect induced by the training protocol.

In addition, MIP increased significantly following the intervention; however, the difference between the size of changes induced by AMT compared to the control group was not significant. Therefore, it is not possible to rule out the potential influence of chance on the size of differences observed between groups.

\section{CONCLUSIONS}

Finally, we may conclude that AMT during eight weeks was enough to increase PEF and respiratory muscle strength, mainly MEP, in sedentary, healthy adolescents. These effects are associated with intervention-induced changes in PCF.

In addition, MIP increased significantly following AMT in the experimental group; however, no differences were observed in the effect size compared to the control group. Future studies are required to confirm whether AMT has an effect on inspiratory muscle strength.

\section{Acknowledgments}

We would like to thank all adolescents and their parents for agreeing to participate in this study and the school for letting us conduct this project.

Also, to the Kinesiology students who helped us with this research line.

\section{REFERENCES}

1. Neve V, Cuisset JM, Edme JL, Carpentier A, et al. Sniff nasal inspiratory pressure in the longitudinal assessment of young Duchenne muscular dystrophy children. Eur Respir J 2013;42(3):671-80.

2. Dassios TG, Katelari A, Doudounakis S, Dimitriou G. Chronic Pseudomonas aeruginosa infection and respiratory muscle impairment in cystic fibrosis. Respir Care 2014;59(3):363-70.

3. Rodríguez I, Arriagada R, Fuentes C, Zenteno D. Aspectos fisiopatológicos de la rehabilitación respiratoria en fibrosis quística. Neumol Pediatr 2012;7(2):51-7.

4. Racca F, DelSorbo L, Mongini T, Vianello A, etal. Respiratory management of acute respiratory failure in neuromuscular diseases. Minerva Anestesiol 2010;76(1):51-62.

4. Koessler W, Wanke T, Winkler G, Nader A, et al. 2 Years' experience with inspiratory muscle training in patients with neuromuscular disorders. Chest 2001;120(3):765-9.

6. Rodríguez I,Zenteno D, Manterola C. Effects of home-based respiratory muscle training in children and adolescents with chronic lung disease. J Bras Pneumol 2014;40(6):626- 33.

7. Rodríguez I, Fuentes C, Rivas C, Molina F, et al. Rehabilitación respiratoria en el paciente neuromuscular: efectos sobre la tolerancia al ejercicio y musculatura respiratoria. Resultado de una serie de casos. Rev Chil Enferm Respir 2013;29(4):196-203.

8. Zenteno D, Puppo H, Vera R, Torres R, et al. Guías de rehabilitación para niños con enfermedades respiratorias crónicas. Neumol Pediatr 2007;3(Supl 1):25-33.

9. Strongoli LM, Gomez CL, Coast JR. The effect of core exercises on transdiaphragmatic pressure. J Sports Sci Med 2010;9(2):270-4.

10. Suzuki J, Tanaka R, Yan S, Chen R, et al. Assessment of abdominal muscle contractility, strength, and fatigue. Am J Respir Crit Care Med 1999;159(4 Pt 1):1052-60.

11. DePalo VA, Parker AL, Al-Bilbeisi F, McCool FD. Respiratory muscle strength training with nonrespiratory maneuvers. J Appl Physiol (1985) 2004;96(2):731-4.

12. Lee BB, Boswell-Ruys C, Butler JE, Gandevia SC. Surface functional electrical stimulation of the abdominal muscles to enhance cough and assist tracheostomy decannulation after high-level spinal cord injury. J Spinal Cord Med 2008;31(1):78-82.

13. McBain RA, Boswell-Ruys CL, Lee BB, Gandevia SC, et al. Abdominal muscle training can enhance cough after spinal cord injury. Neurorehabil Neural Repair 2013;27(9):834-43.

14. Rodríguez I, Alarcón M, Gutierrez C, Hermosilla P, et al. Efecto del entrenamiento de músculos abdominales sobre la función respiratoria en adolescentes sanos: Estudio piloto. Rev Chil Enferm Respir 2014;30(4):203-11.

15. American Thoracic Society/European Respiratory Society. ATS/ERS Statement on respiratory muscle testing. Am J Respir Crit Care Med 2002;166(4):518-624.

16. Céspedes J, Gutiérrez M, Oyarzún M. Flujometría en la práctica de atención primaria. Rev Chil Enferm Respir 2010;26(1):47-8. 
17. Rodríguez I. Confiabilidad de la fuerza muscular respiratoria y flujos espiratorios forzados en adolescentes sanos. Rev Chil Enferm Respir 2015;31(2):86-93.

18. Elbasan B, Tunali N, Duzgun I, Ozcelik U. Effects of chest physiotherapy and aerobic exercise training on physical fitness in young children with cystic fibrosis. Ital J Pediatr 2012;38:2.

19. Illi SK, Held U, Frank I, Spengler CM. Effect of respiratory muscle training on exercise performance in healthy individuals: a systematic review and meta-analysis. Sports Med 2012;42(8):707-24.

20. HajGhanbari B, Yamabayashi C, Buna TR, Coelho JD, et al. Effects of respiratory muscle training on performance in athletes: a systematic review with meta-analyses. JStrength Cond Res 2013;27(6):1643-63.

21. Gollee H, Hunt KJ, Allan DB, Fraser MH, et al. Automatic electrical stimulation of abdominal wall muscles increases tidal volume and cough peak flow in tetraplegia. Technol Health Care 2008;16(4):273-81.

22. McCool FD. Global physiology and pathophysiology of cough: ACCP evidence-based clinical practice guidelines. Chest 2006;129(1 Suppl):48s-53s.

23. Moran F, Piper A, Elborn JS, Bradley JM. Respiratory muscle pressures in non-CF bronchiectasis: repeatability and reliability. Chron Respir Dis 2010;7(3):165-71. 